\title{
Sexual risk compensation following voluntary medical male circumcision: Results from a prospective cohort study amongst human immunodeficiency virus-negative adult men in Botswana
}

\begin{tabular}{|c|c|}
\hline \multicolumn{2}{|c|}{$\begin{array}{l}\text { Authors: } \\
\text { Lisa P. Spees } \\
\text { Kathleen E. Wirth } \\
\text { Shreshth Mawandia } \\
\text { Semo Bazghina-werq } \\
\text { Jenny H. Ledikwe } \\
\text { Jent, }\end{array}$} \\
\hline \multicolumn{2}{|c|}{$\begin{array}{l}\text { Affiliations: } \\
{ }^{1} \text { Department of Health Policy } \\
\text { and Management, Gillings } \\
\text { School of Global Public } \\
\text { Health, University of North } \\
\text { Carolina at Chapel Hill, } \\
\text { Chapel Hill, NC, United States } \\
\text { of America }\end{array}$} \\
\hline \multicolumn{2}{|c|}{$\begin{array}{l}{ }^{2} \text { Lineberger Comprehensive } \\
\text { Cancer Center, University of } \\
\text { North Carolina, Chapel Hill, } \\
\text { NC, United States of America }\end{array}$} \\
\hline \multicolumn{2}{|c|}{$\begin{array}{l}{ }^{3} \text { Department of Biostatistics, } \\
\text { Faculty of Health Sciences, } \\
\text { Harvard T.H. Chan School of } \\
\text { Public Health, Boston, MA, } \\
\text { United States of America }\end{array}$} \\
\hline \multicolumn{2}{|c|}{$\begin{array}{l}{ }^{4} \text { Botswana International } \\
\text { Training and Education } \\
\text { Center for Health (I-TECH), } \\
\text { Gaborone, Botswana }\end{array}$} \\
\hline \multicolumn{2}{|c|}{$\begin{array}{l}{ }^{5} \text { Department of Health Policy } \\
\text { and Management, Faculty of } \\
\text { Medicine, Botswana } \\
\text { International Training and } \\
\text { Education Center for Health, } \\
\text { Gaborone, Botswana }\end{array}$} \\
\hline \multicolumn{2}{|c|}{$\begin{array}{l}{ }^{6} \text { Department of Global } \\
\text { Health, University of } \\
\text { Washington, Seattle, WA, } \\
\text { United States of America }\end{array}$} \\
\hline \multicolumn{2}{|c|}{$\begin{array}{l}{ }^{7} \text { Independent Global Health } \\
\text { Consultant, Washington, DC, } \\
\text { United States of America }\end{array}$} \\
\hline \multicolumn{2}{|l|}{ Read online: } \\
\hline 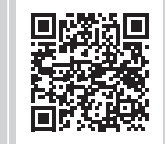 & $\begin{array}{l}\text { Scan this QR } \\
\text { code with your } \\
\text { smart phone or } \\
\text { mobile device } \\
\text { to read online. }\end{array}$ \\
\hline
\end{tabular}

Background: Circumcised men may increase sexual risk-taking following voluntary medical male circumcision (VMMC) because of decreased perceptions of risk, which may negate the beneficial impact of VMMC in preventing new human immunodeficiency virus (HIV) infections.

Objectives: We evaluated changes in sexual behaviour following VMMC.

Method: We conducted a prospective cohort study amongst sexually active, HIV-negative adult men undergoing VMMC in Gaborone, Botswana, during 2013-2015. Risky sexual behaviour, defined as the number of sexual partners in the previous month and $\geq 1$ concurrent sexual partnerships during the previous 3 months, was assessed at baseline (prior to VMMC) and 3 months post-VMMC. Change over time was assessed by using inverse probability weighted linear and conditional logistic regression models.

Results: We enrolled 523 men; 509 (97\%) provided sexual behaviour information at baseline. At 3 months post-VMMC, 368 (72\%) completed the follow-up questionnaire. At baseline, the mean $(95 \%$ confidence interval) number of sexual partners was $1.60(1.48,1.65)$, and $111(31 \%$ of 353 with data) men reported engaging in concurrent partnerships. At 3 months post-VMMC, 70 (23\% of 311 with data) reported fewer partners and 19\% had more partners. Amongst 111 men with a concurrent partnership at baseline, 52\% reported none post-VMMC. Amongst the $242(69 \%)$ without a concurrent partnership at baseline, 19\% reported initiating one postVMMC. After adjustment for loss to follow-up, risky sexual behaviour post-VMMC (measured as mean changes in a number of partners and proportion engaging in concurrency) was similar to baseline levels.

Conclusion: We found no evidence of sexual risk compensation in the 3 months following VMMC.

Keywords: circumcision; HIV prevention; Botswana; sexual behaviour; risk-taking; prospective studies.

\section{Introduction}

Three randomised controlled trials (RCTs) from sub-Saharan Africa showed that voluntary medical male circumcision (VMMC) reduced human immunodeficiency virus (HIV) transmission from females to males by up to $60 \%{ }^{1,2,3}$ Mathematical models suggest that HIV incidence will decrease in circumcised men, and subsequently in women and uncircumcised men, as the uptake of VMMC increases. ${ }^{4,5,6}$ Botswana has the fourth highest HIV prevalence in the world, with one in five adults infected. ${ }^{7}$ Whilst $83 \%$ HIV-positive individuals are receiving treatment, HIV incidence remains high. ${ }^{7}$ In 2018, 8500 new HIV infections were diagnosed. ${ }^{7}$ In Botswana, it is estimated that expanding VMMC coverage would avert 4900 new HIV infections through $2030 .^{8}$

There are concerns that circumcised men may be more likely to engage in risky sexual behaviours following surgery because of their perception of the reduced risk of HIV acquisition conferred

\footnotetext{
Corresponding author: Lisa Spees, Ispees21@live.unc.edu
}

Dates: Received: 19 Aug. 20 | Accepted: 21 Oct. 20 | Published: 14 Dec. 2020

How to cite this article: Spees LP, Wirth KE, Mawandia S, Bazghina-werq S, Ledikwe JH. Sexual risk compensation following voluntary medical male circumcision: Results from a prospective cohort study amongst human immunodeficiency virus-negative adult men in Botswana. S Afr J HIV Med. 2020;21(1), a1157. https://doi.org/10.4102/sajhivmed.v21i1.1157

Copyright: ( 2020. The Authors. Licensee: AOSIS. This work is licensed under the Creative Commons Attribution License. 
by circumcision. This phenomenon, known as risk compensation or behaviour disinhibition, may reduce the effectiveness of VMMC in preventing new HIV infections. Although RCTs conducted in Kenya and Uganda indicated that risk compensation behaviour did not increase following circumcision, ${ }^{9}$ a RCT conducted in South Africa documented a higher number of sexual contacts amongst circumcised men compared with uncircumcised men in the control group. ${ }^{1}$ However, amongst recent cross-sectional surveys conducted in South Africa, Uganda and Kenya, there was no evidence that circumcised men's behaviour was riskier than uncircumcised men's behaviour. ${ }^{10,11,12}$

Men circumcised in non-clinical trial settings may also encounter different experiences and behave differently. However, little is known about how sexual behaviour changes in real-world settings, as there have been only two studies that examined men's sexual behaviours before and after undergoing VMMC. ${ }^{13,14}$ Although the two studies, both conducted in South Africa, found minimal or no evidence of risk compensation, no studies have examined risk compensation in Botswana, where the rate of multiple and concurrent sexual partnerships is particularly high. Recently published findings from a large population-based sample found that $31 \%$ of sexually active adults in Botswana had concurrent sexual partners in the past year. ${ }^{15}$

We conducted a prospective cohort study amongst men aged 18-49 years undergoing VMMC in Botswana to evaluate the changes, if any, in the frequency of high-risk sexual behaviour following VMMC, including the total number and timing of sexual partnerships during the previous 3 months.

\section{Methods}

\section{Study design}

This clinic-based prospective cohort study was designed to assess (1) the frequency, type and severity of adverse events immediately following VMMC; (2) the prevalence and correlates of re-initiation of sexual activity and (3) changes in risky sexual behaviour following VMMC. The current analysis reports on the third primary objective. The study was conducted by the International Training and Education Center for Health (I-TECH), a collaboration with the University of Washington and the University of California, San Francisco. Recruitment and enrolment of study participants occurred before undergoing VMMC, but after individuals completed group education and individual counselling with clinic staff (including HIV testing) and provided written, informed consent for the procedure. Neither the pre-procedure activities described above nor the procedure itself was performed by study staff. The overall objective of Botswana's National Safe Male Circumcision programme is to reach a male circumcision prevalence rate of $80 \%$ amongst $0-49$-year-old HIV-negative males. A complete description of the programme, including details on the procedure itself, can be found elsewhere. ${ }^{16}$

\section{Study setting and participants}

Adult men undergoing VMMC through the National Safe Male Circumcision programme were enrolled between November 2013 and April 2015 at two government-run clinics providing free circumcision services in Gaborone, the capital city of Botswana. Participant eligibility criteria included: age ranging 18-49 years, residence within $25 \mathrm{~km}$ of Gaborone, ever had sexual intercourse and documented HIV-negative test result. All participants provided written informed consent for participation in the study in addition to the consent obtained by clinic staff for the circumcision procedure.

\section{Sample size}

For study planning purposes, we computed sample size requirements (and corresponding power) based on the dichotomous outcome and engagement in concurrent sexual partnership(s) during the previous 3 months. We used McNemar's test for two correlated proportions to determine the number of participants enrolled and the corresponding power to detect the smallest, clinically meaningful difference in the proportion of men who report engaging in a concurrent sexual partnership between baseline and 3 months post-circumcision. ${ }^{17}$ Figure 1 shows the summary of the proportions associated with all possible responses under this framework.

Specifically, $\mathrm{P}_{\mathrm{B}}$ and $\mathrm{P}_{\mathrm{FU}}$ represent the total proportion of individuals reporting high-risk sexual behaviour at baseline and follow-up, respectively; $\mathrm{P}_{01}$ and $\mathrm{P}_{10}$ represent the proportion of individuals who increase or decrease their high-risk sexual behaviour between baseline and follow-up, respectively. We explicitly chose to test a one-sided alternative that high-risk sexual behaviour increases at follow-up (as opposed to the two-sided alternative of any change) to reflect the conceptual definition of risk compensation. Thus, assuming a baseline prevalence of sexual concurrency $\left(\mathrm{P}_{\mathrm{B}}\right)$ of $19 \%, 18 \%$ were unable to undergo VMMC because of medical or other contraindications and $20 \%$ were lost to

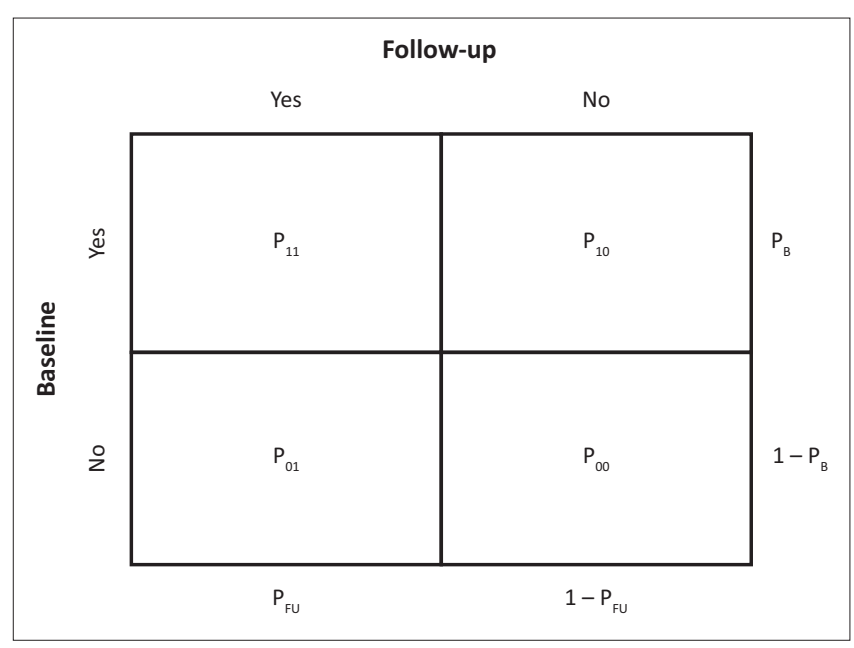

FIGURE 1: Contingency table used for calculating power based on McNemar's test of two correlated proportions. 
follow-up, power was estimated to be $>80 \%$ to detect a $5 \%$ increase in the proportion of men who engage in high-risk sexual behaviour at 3 months post-circumcision (relative to baseline) based on a sample size of 523 participants. Power was estimated to be $99 \%$ for a $10 \%$ increase at 3 months post-VMMC.

\section{Measures}

In addition to the primary endpoints, the baseline questionnaire also collected information on demographics (age, current relationship status and religious affiliation), socioeconomic indicators (education, employment status, electricity in household, refrigerator in household and use of wood as cooking fuel), frequency and intensity of alcohol consumption, age at sexual initiation and history of transactional sex. We also collected information on the primary reason for getting circumcised (i.e. protecting against HIV, personal hygiene or other), whether the participant believed circumcision would have a negative, positive or no impact on the quality of sex, and administered a fourquestion scale developed in South Africa to assess attitudes towards condom use, monogamy and HIV risk in the context of circumcision. ${ }^{19}$ Lastly, we constructed two indicators evaluating the accuracy of participants' knowledge regarding the effect of VMMC on female-to-male and male-to-female HIV transmission. Specifically, we classified men as having correct knowledge if they indicated that VMMC partially protects a man from getting HIV from a woman but does not affect a woman's chances of getting HIV from a man.

\section{Data collection}

Before undergoing circumcision, all study participants were asked to complete a baseline sexual behaviour questionnaire through an audio computer-assisted self-interviewing (ACASI) tool in a private office room at the study site. This interviewing technique circumvented Social Desirability bias by allowing the participant to complete the questionnaire in a standardised format without having to share their responses with study personnel directly. However, if the participant was unable or preferred not to use the ACASI tool, the study nurse was available to administer the questionnaire. The tool, available in both English and the local language, Setswana, was developed by using Questionnaire Development System $\left(\right.$ QDS $\left.^{\mathrm{TM}}\right)$ questionnaire and survey development software adapted for low literacy and computer naïve populations (Nova Research Company, Silver Spring, MD).

After circumcision, post-procedure visits at 2 days, 7 days, 6 weeks and 3 months were scheduled as outlined by the Botswana Ministry of Health $(\mathrm{MOH})$ guidelines for adult VMMC. At each visit, participants completed the follow-up survey through ACASI during their clinic appointment. A follow-up visit at 12 months was planned to coincide with annual HIV testing as per standard of care in Botswana. Each study participant was provided with a wallet-size reminder card noting the date of each follow-up visit.
Participants received BWP100 (approximately USD\$8 at study initiation) at each post-operative visit as compensation for their time and travel costs.

The current analysis is restricted to data collected at baseline and the 3-month follow-up visit when the sexual behaviour questionnaire was repeated. Study data were collected and managed by using Research Electronic Data Capture (REDCap), a secure, web-based application designed to support data capture for research studies hosted at the Institute for Translational Health Sciences at the University of Washington. ${ }^{20}$

\section{Outcomes}

The primary outcomes of the current analysis were (1) the number of sexual partners in the previous 1 month and (2) one or more concurrent sexual partnerships during the previous 3 months. In accordance with the Joint United Nations Programme on HIV/AIDS (UNAIDS) recommendations, we defined a concurrent sexual partnership as 'overlapping sexual partnerships in which sexual intercourse with one partner occurs between two acts of intercourse with another partner'. ${ }^{21}$ Concurrency was then assessed by using the following threequestions (based onUNAIDSrecommendations for data collection), each of which was asked of participant's three most recent sexual partners: 'How long ago did you first have sexual intercourse with this person?' 'When was the last time you had sexual intercourse with this person?' and 'Are you still having sex with this person?'

To evaluate the change in the number of sexual partners and engagement in concurrent sexual partnerships before and after undergoing VMMC, we constructed change scores for each endpoint, which subtracted participants' baseline response from that reported at the 3-month follow-up interview.

\section{Statistical analyses}

For the continuous outcome, the number of sexual partners (past 1 month), we fit an intercept-only inverse-probability weighted linear regression model to estimate the mean change in the number of partners at 3 months post-VMMC (compared to baseline). For the dichotomous outcome, engagement in concurrent sexual partnerships (past 3 months), we fit an inverse-probability weighted conditional logistic regression model (stratified on the participant) to estimate the change in the proportion of participants reporting sexual concurrency at 3 months post-VMMC (compared with baseline).

Inverse probability weighting was used to adjust for potential selection bias because of non-trivial (anticipated to be $20 \%$ before study start) loss to follow-up..$^{22,23,24}$ Inverse-probability weighting adjusts for loss to follow-up by empirically breaking the association between observed predictors (collected at baseline) and participation at follow-up, allowing for unbiased estimation in the weighted sample, 
provided a regression for participation is correctly specified and no unobserved correlates of non-participation and risky sexual behaviour exist. Inverse probability weights for participation at follow-up were constructed from a multivariable logistic regression model, which considered the following 24 potential covariates based on subject-matter knowledge: age, relationship status, religious affiliation, education, employment status, household assets or characteristics, reason for circumcision, correct knowledge of circumcision benefits, beliefs about circumcision, alcohol use, age at first sex, number of sexual partners (past 1 month, 1 year and lifetime) and transactional sex. We also created 117 two-way interaction terms by taking the cross-product of each demographic and socioeconomic covariates with each knowledge, belief and behavioural covariate. To build the multivariate logistic regression model required by inverseprobability weighting, we used a stepwise, forward selection procedure to identify covariates from the list of candidate predictors listed above. The entry and exit criteria were set to a $p<0.2$. We included missing indicators for each selected variable to maximise the number of cases included in the final models and to maintain a constant sample size across analyses.

In post-hoc analyses, we sought to identify attitudes, beliefs and/or behaviours reported at baseline that may be predictive of engagement (irrespective of what the participant reported at baseline [i.e. pre-circumcision]) in high-risk sexual behaviour at 3 months post-VMMC. Specifically, we fit separate univariable- and multivariable-adjusted modified Poisson regression models (weighted by the inverse of the probability of participation at follow-up) for each of our primary outcomes (with the number of sexual partners in the past 1 month dichotomised at two or more) for each of the following covariates: alcohol consumption, reasons for circumcision, correct knowledge of circumcision benefits, beliefs about circumcision, age at first sex and transactional sex. All multivariable models were adjusted for potential confounding by the following demographic and socioeconomic covariates (all assessed at baseline): age, relationship status, religious affiliation, education, employment and household use of wood as cooking fuel.

All analyses were conducted by using SAS software version 9.4 (SAS Institute, Cary, NC).

\section{Ethical consideration}

Ethical approvals were obtained from the Health Research and Development Committee at the Botswana Ministry of Health (MOH) (\#00699) and the University of Washington Institutional Review Board (\#42047).

\section{Results}

Between November 2013 and October 2015, research staff screened 577 men preparing to undergo VMMC for study participation (Figure 2). A total of 528 (92\%) participants were determined to be eligible for participation and 523 (91\%) subsequently enrolled. Reasons for ineligibility

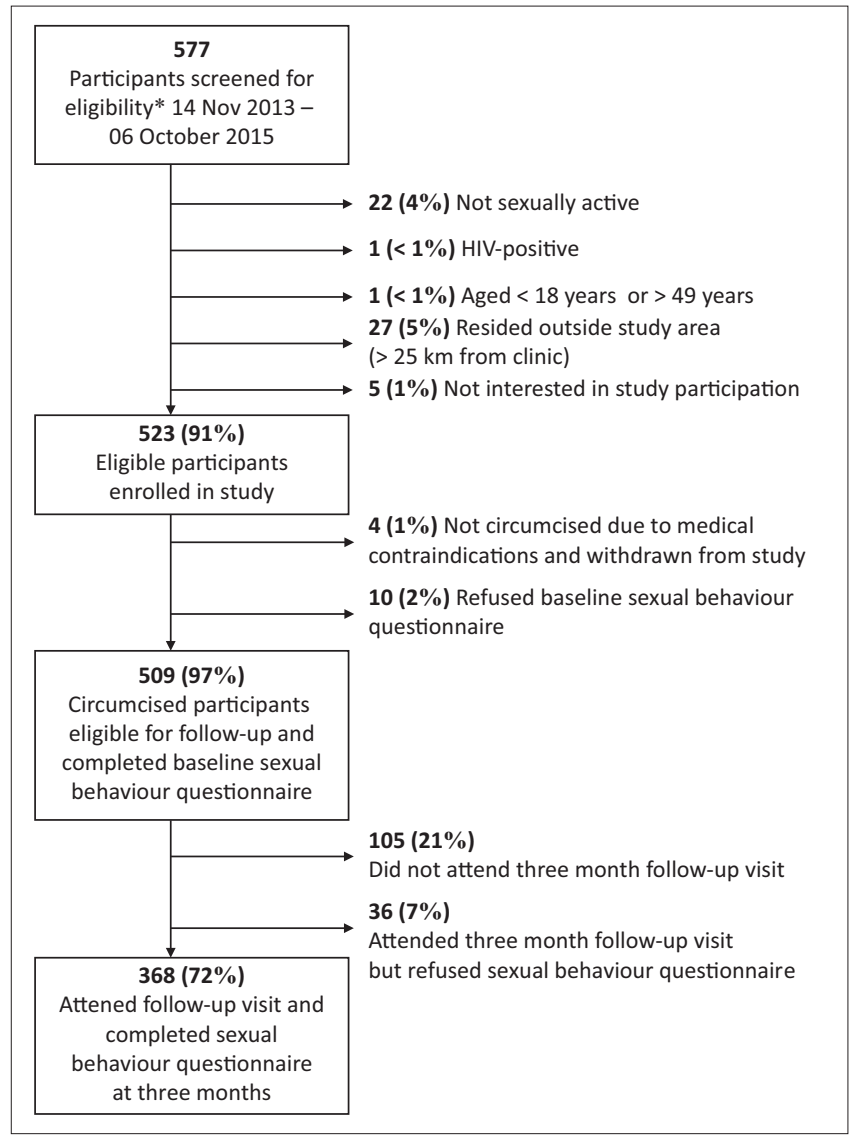

Note: *, Two particpants did not meet two eligibility vriteria. Therefore the number and percentages presented for invidual reasons for ineligibility will sum to $>100 \%$.

FIGURE 2: Consolidated Standards of Reporting Trials (CONSORT) diagram illustrating screening, eligibility, enrolment and follow-up of men undergoing voluntary medical male circumcision in Gaborone, Botswana, 2013-2015.

included not sexually active $(4 \%)$, residence outside of the area $(5 \%)$, HIV infection $(<1 \%)$ and age $<18$ years or $>49$ years $(<1 \%)$. Four individuals who met study eligibility criteria and consented to study participation were not circumcised because of medical contraindications that were identified before the procedure. Amongst the 509 circumcised participants who completed the baseline sexual behaviour questionnaire, 368 (72\%) attended the follow-up visit and completed the sexual behaviour questionnaire at 3 months post-circumcision. Because of missing data on specified outcomes, 353 were included in the analysis examining the number of sexual partners in the past month, and 311 were included in the sexual concurrency analysis.

Table 1 shows the summary of the baseline characteristics of the study population according to the availability of sexual behaviour data at 3 months post-circumcision. Men who either did not complete the follow-up visit $(n=105)$ or refused the sexual behaviour questionnaire $(n=36)$ were more highly educated (odds ratio [OR]: 1.72; 95\% confidence interval [CI]: 1.13-2.61) and underwent circumcision for personal hygiene reasons (OR: 1.66; 95\% CI: 1.07-2.57) compared with respondents at 3 months post-VMMC. In contrast, nonrespondents were less likely to live in larger households (OR: 0.59; 95\% CI: 0.36-0.97). No other baseline characteristics were significantly associated with the response at 3 months post-VMMC. 
TABLE 1: Distribution of baseline characteristics of $N=509$ HIV-negative, sexually active adult men who underwent voluntary medical male circumcision according to the availability of sexual behaviour data at 3 months post-voluntary medical male circumcision and association of these characteristics with data availability.

\begin{tabular}{|c|c|c|c|c|c|c|}
\hline \multirow[t]{3}{*}{ Baseline characteristics ( $n$ with data) } & \multicolumn{6}{|c|}{ Sexual behaviour data at $\mathbf{3}$ months Post-VMMC, $\uparrow$} \\
\hline & \multicolumn{2}{|c|}{ Available $(n=368)$} & \multicolumn{2}{|c|}{ Not available $(n=141)$} & \multirow[t]{2}{*}{ OR } & \multirow[t]{2}{*}{$95 \% \mathrm{Cl}$} \\
\hline & $N$ & $\%$ & $N$ & $\%$ & & \\
\hline \multicolumn{7}{|l|}{ Demographic } \\
\hline \multicolumn{7}{|l|}{ Age $(n=509)$ (years) } \\
\hline $18-24$ & 110 & 30 & 53 & 38 & 1 & ref. \\
\hline $25-29$ & 123 & 33 & 50 & 36 & 0.84 & $0.53-1.34$ \\
\hline $35-49$ & 59 & 16 & 16 & 11 & 0.56 & $0.30-1.07$ \\
\hline \multicolumn{7}{|l|}{ Relationship status ( $n=509$ ) } \\
\hline Single‡ & 71 & 19 & 26 & 18 & 1 & ref. \\
\hline Dating and living together & 72 & 20 & 24 & 17 & 0.91 & $0.48-1.73$ \\
\hline Dating but not living together & 196 & 53 & 76 & 54 & 1.06 & $0.63-1.78$ \\
\hline Married & 29 & 8 & 15 & 11 & 1.41 & $0.66-3.05$ \\
\hline \multicolumn{7}{|l|}{ Religious affiliation ( $n=508$ ) } \\
\hline Any religious affiliation & 346 & 94 & 136 & 96 & 1.65 & $0.61-4.47$ \\
\hline \multicolumn{7}{|l|}{ Socioeconomic } \\
\hline \multicolumn{7}{|l|}{ Highest level of education completed $(n=492) \S$} \\
\hline Secondary or less & 168 & 47 & 44 & 34 & 1 & ref. \\
\hline Higher than secondary & 193 & 54 & 87 & 66 & 1.72 & $1.13-2.61$ \\
\hline Employed or self-employed $(n=509)$ & 263 & 72 & 96 & 68 & 0.85 & $0.56-1.30$ \\
\hline \multicolumn{7}{|l|}{ Household size $(n=507)$} \\
\hline $0-2$ & 98 & 27 & 52 & 37 & 1 & ref. \\
\hline $3-4$ & 124 & 34 & 39 & 28 & 0.59 & $0.36-0.97$ \\
\hline $5-6$ & 79 & 22 & 31 & 22 & 0.74 & $0.43-1.26$ \\
\hline$\geq 7$ & 65 & 18 & 19 & 14 & 0.55 & $0.30-1.02$ \\
\hline Electricity in household $(n=509)$ & 314 & 85 & 121 & 86 & 1.04 & $0.60-1.81$ \\
\hline Refrigerator in household $(n=509$ ) & 282 & 77 & 112 & 79 & 1.18 & $0.73-1.89$ \\
\hline Household use of wood as cooking fuel $(n=509)$ & 61 & 17 & 23 & 16 & 0.98 & $0.58-1.66$ \\
\hline HIV protection & 180 & 49 & 61 & 43 & 1 & ref. \\
\hline Personal hygiene & 98 & 27 & 55 & 39 & 1.66 & $1.07-2.57$ \\
\hline Other & 90 & 25 & 25 & 18 & 0.82 & $0.48-1.39$ \\
\hline \multicolumn{7}{|l|}{ Knowledge of HIV risk } \\
\hline Correct knowledge: VMMC partially reduces HIV risk for men $(n=509)$ & 311 & 85 & 112 & 79 & 0.71 & $0.43-1.16$ \\
\hline Correct knowledge: VMMC does not impact HIV risk for women $(n=506)$ & 40 & 11 & 15 & 11 & 0.97 & $0.52-1.81$ \\
\hline \multicolumn{7}{|l|}{ Risk compensation scale, mean (SD) } \\
\hline Condom use is not necessary if the man is circumcised $(n=509)$ & 4.35 & 1.00 & 4.28 & 1.26 & 0.94 & $0.79-1.12$ \\
\hline If I am circumcised, sex is safe without a condom $(n=509)$ & 4.42 & 0.90 & 4.35 & 1.13 & 0.94 & $0.77-1.14$ \\
\hline Being circumcised means a man can worry less about HIV ( $n=508)$ & 3.79 & 1.46 & 3.91 & 1.42 & 1.06 & $0.93-1.22$ \\
\hline If a man is circumcised, he can have more sexual partners $(n=509)$ & 4.51 & 0.73 & 4.47 & 1.03 & 0.95 & $0.75-1.19$ \\
\hline Behavioural & & & & & & \\
\hline Alcohol consumption ( $n=509$ ) & 224 & 61 & 88 & 62 & 1.07 & $0.72-1.59$ \\
\hline Age at first sexual intercourse $(n=501)$ (years) & & & & & & \\
\hline$<18$ & 104 & 28 & 45 & 32 & 1 & ref. \\
\hline $18-20$ & 164 & 45 & 65 & 46 & 1.09 & $0.69-1.72$ \\
\hline$\geq 21$ & 93 & 25 & 31 & 22 & 1.40 & $0.82-2.38$ \\
\hline$\geq 2$ partners, past 1 month $(n=461)$ & 148 & 44 & 59 & 47 & 1.11 & $0.74-1.68$ \\
\hline$\geq 2$ partners, past 12 months $(n=466)$ & 201 & 60 & 78 & 61 & 1.06 & $0.70-1.61$ \\
\hline Number of partners, lifetime $(n=505)$ & & & & & & \\
\hline 1 partner & 33 & 9 & 18 & 13 & 1 & ref. \\
\hline 2-4 partners & 150 & 41 & 52 & 37 & 0.64 & $0.33-1.22$ \\
\hline 5-10 partners & 122 & 33 & 47 & 34 & 0.71 & $0.36-1.37$ \\
\hline 11 or more partners & 60 & 16 & 23 & 16 & 0.71 & $0.33-1.49$ \\
\hline Exchanged money for sex, past 12 months $(n=468)$ & 23 & 7 & 10 & 8 & 1.17 & $0.54-2.53$ \\
\hline
\end{tabular}

VMMC, voluntary medical male circumcision; OR, odds ratio; HIV, human immunodeficiency virus; SD, standard deviation.

$\dagger$, Data are $n$ (column \%) unless otherwise noted. Values may not total to $100 \%$ because of rounding.

$\$$, Category includes single, separated, divorced and widowed participants.

$\$$, Category includes participants reporting no school attendance, non-formal schooling, primary education and secondary education.

ๆ, Median (25th, 75th percentile) of Likert scale (range $0-5$ with 0 and 5 denoting 'strongly disagree' and 'strongly agree', respectively). 
Figure 3 shows the summary of the change in the number of sexual partners and engagement in concurrent sexual partnerships at the 3-month follow-up visit (relative to baseline). Amongst the 368 men who attended the follow-up visit at 3 months, 57 (15\%) did not provide information on the number of partners during the past 1 month at either baseline and/or follow-up. Data on engagement in concurrent sexual partnerships were available for 353 (96\%) of the 368 men who attended follow-up.

At baseline, the mean $(95 \% \mathrm{CI})$ number of sexual partners (past 1 month) was $1.60(1.48,1.65)$. Although the majority (59\% of $n=311$ with available data) of men reported the same number of partners at 3 months post-VMMC, $70(23 \%)$

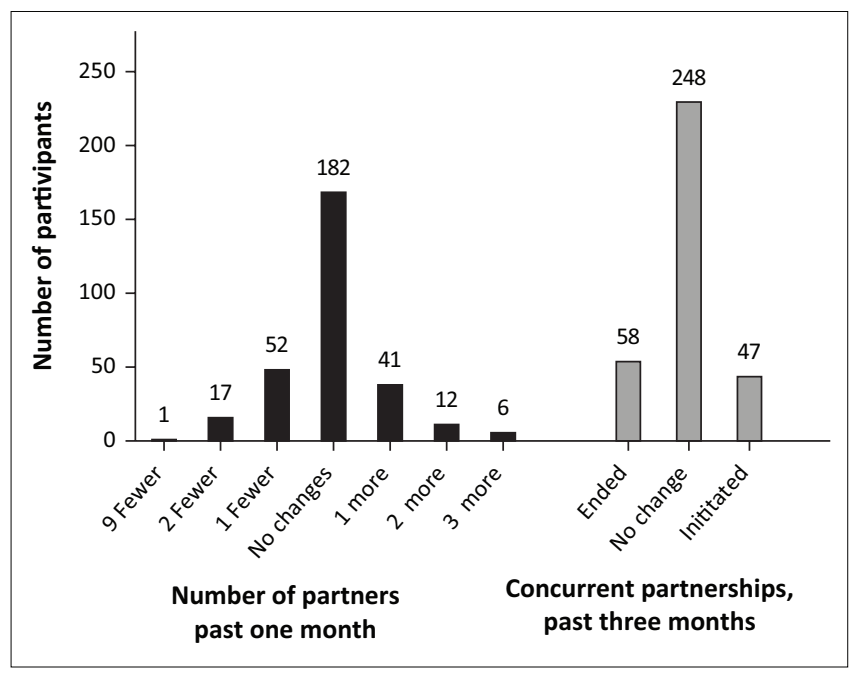

FIGURE 3: Summary of change in the number of sexual partners (past 1 month) and engagement in concurrent partnerships at 3 months amongst men undergoing voluntary medical male circumcision in Gaborone, Botswana, 2012-2015. reported fewer partners and $59(19 \%)$ had more partners (Figure 3). For concurrency (past 3 months), at baseline, 111 (31\% of $n=353$ with available data) reported engaging in one or more concurrent sexual partnerships. By 3 months post-VMMC, 58 (52\% of 111) had ended the concurrent partnership. In contrast, amongst the $242(69 \%)$ who did not report any concurrent sexual partnerships at baseline, $47(19 \%$ of 242$)$ had initiated a concurrent partnership at 3 months (Figure 3). After adjustment for possible selection bias because of loss to follow-up at 3 months, we found no evidence of sexual risk compensation following VMMC: mean change in number of partners was -0.04 (95\% CI: -0.15 , $0.08 ; p=0.61$ ) and the proportion engaging in concurrency was 0.04 (95\% CI: $-0.05,0.14 ; p=0.38)$.

Tables 2 and 3 present the results of post-hoc analyses aimed at identifying predictors of any engagement (irrespective of change from baseline or pre-circumcision) in high-risk sexual behaviour at 3 months post-VMMC. In multivariableadjusted models (simultaneously adjusted for potential selection bias because of loss to follow-up), alcohol consumption (Table 2; risk ratio [RR]: 1.72; 95\% CI: 1.19, 2.49) and transactional sex (RR: 1.77; 95\% CI: 1.13, 2.77) were positively associated with reporting two or more partners (past 1 month) at follow-up. Alcohol consumption was also positively associated with concurrency at 3 months postVMMC (Table 3; RR: 2.30; 95\% CI: 1.45, 3.66). In contrast, older age at first sexual intercourse (21 years and older) was negatively associated with multiple sexual partners (Table 2; RR: 0.50; 95\% CI: 0.30, 0.84) at follow-up. No other attitudinal or behavioural covariates were significantly associated with either outcome in either univariable- or multivariableadjusted analyses.

TABLE 2: Univariable- and multivariable-adjusted attitudinal and behavioural predictors of multiple sexual partners (past 1 month) at 3 months amongst men undergoing voluntary medical male circumcision in Gaborone, Botswana, 2012-2015.

\begin{tabular}{|c|c|c|c|c|c|c|}
\hline \multirow[t]{2}{*}{ Attitudinal and behavioural predictors } & \multicolumn{3}{|c|}{ Univariable-adjusted $\dagger$} & \multicolumn{3}{|c|}{ Multivariable-adjusted $\uparrow, *$} \\
\hline & $\mathbf{R R}$ & $95 \% \mathrm{Cl}$ & $p$ & $\mathbf{R R}$ & $95 \% \mathrm{Cl}$ & $p$ \\
\hline \multicolumn{7}{|l|}{ Reasons for circumcision } \\
\hline Personal hygiene & 1 & ref. & - & 1 & ref. & - \\
\hline HIV protection & 0.96 & $0.68-1.35$ & 0.80 & 0.99 & $0.70-1.40$ & 0.96 \\
\hline Other & 0.90 & $0.60-1.35$ & 0.61 & 0.86 & $0.58-1.28$ & 0.45 \\
\hline \multicolumn{7}{|l|}{ Knowledge of HIV risk } \\
\hline Correct knowledge: VMMC partially reduces HIV risk for men & 1.12 & $0.71-1.77$ & 0.62 & 1.15 & $0.73-1.83$ & 0.54 \\
\hline Correct knowledge: VMMC does not impact HIV risk for women & 1.14 & $0.73-1.78$ & 0.55 & 1.13 & $0.71-1.80$ & 0.60 \\
\hline \multicolumn{7}{|l|}{ Risk compensation scale } \\
\hline Condom use is not necessary if the man is circumcised & 1.02 & $0.86-1.21$ & 0.80 & 1.04 & $0.86-1.25$ & 0.69 \\
\hline If I am circumcised, sex is safe without a condom & 0.99 & $0.85-1.17$ & 0.95 & 0.97 & $0.82-1.14$ & 0.70 \\
\hline Being circumcised means a man can worry less about HIV & 1.03 & $0.92-1.14$ & 0.63 & 1.05 & $0.94-1.17$ & 0.36 \\
\hline If a man is circumcised, he can have more sexual partners & 1.10 & $0.87-1.39$ & 0.42 & 1.08 & $0.86-1.37$ & 0.49 \\
\hline Alcohol consumption & 1.80 & $1.23-2.62$ & 0.002 & 1.80 & $1.23-2.62$ & 0.004 \\
\hline$<18$ & 1 & ref. & - & 1 & ref. & - \\
\hline $18-20$ & 0.98 & $0.72-1.34$ & 0.92 & 0.92 & $0.68-124$ & 0.59 \\
\hline$\geq 21$ & 0.51 & $0.30-0.85$ & 0.01 & 0.50 & $0.30-0.84$ & 0.01 \\
\hline Exchanged money for sex, past 12 months & 1.64 & $1.07-2.52$ & 0.02 & 1.77 & $1.13-2.77$ & 0.01 \\
\hline
\end{tabular}

$\mathrm{RR}$, risk ratio; $\mathrm{Cl}$, confidence intervals; HIV, human immunodeficiency virus; VMMC, voluntary medical male circumcision.

$\dagger$, Estimated from a weighted modified Poisson regression model with weights constructed to adjust for selection bias because of loss to follow-up.

\$, Estimated from a weighted modified Poisson regression model with weights constructed to adjust for selection bias because of loss to follow-up and confounding because of the following baseline covariates: age, relationship status, religious affiliation, education, employment and household use of woods as cooking fuel.

$\S$, Responses based on Likert scale (range $0-5$ with 0 and 5 denoting 'strongly disagree' and 'strongly agree', respectively). 
TABLE 3: Univariable- and multivariable-adjusted attitudinal and behavioural predictors of concurrent sexual partnerships (past 3 months) at 3 months amongst men undergoing voluntary medical male circumcision in Gaborone, Botswana, 2012-2015.

\begin{tabular}{|c|c|c|c|c|c|c|}
\hline \multirow[t]{2}{*}{ Attitudinal and behavioural predictors } & \multicolumn{3}{|c|}{ Univariable-adjusted $\dagger$} & \multicolumn{3}{|c|}{ Multivariable-adjusted $\dagger, *$} \\
\hline & $\mathbf{R R}$ & $95 \% \mathrm{Cl}$ & $p$ & $\mathbf{R R}$ & $95 \% \mathrm{Cl}$ & $p$ \\
\hline \multicolumn{7}{|l|}{ Reasons for circumcision } \\
\hline Personal hygiene & 1 & ref. & - & 1 & ref. & - \\
\hline HIV protection & 1.16 & $0.74-1.82$ & 0.52 & 1.24 & $0.79-1.96$ & 0.35 \\
\hline Other & 0.98 & $0.57-1.67$ & 0.94 & 0.91 & $0.55-1.53$ & 0.74 \\
\hline \multicolumn{7}{|l|}{ Knowledge of HIV risk } \\
\hline Correct knowledge: VMMC reduces HIV risk for men & 0.99 & $0.58-1.68$ & 0.97 & 1.05 & $0.59-1.86$ & 0.87 \\
\hline Correct knowledge: VMMC does not impact HIV risk for women & 1.23 & $0.71-2.10$ & 0.46 & 1.16 & $0.66-2.01$ & 0.61 \\
\hline \multicolumn{7}{|l|}{ Risk compensation scale } \\
\hline Condom use is not necessary if the man is circumcised & 1.13 & $0.91-1.40$ & 0.29 & 1.18 & $0.93-1.49$ & 0.17 \\
\hline If I am circumcised, sex is safe without a condom & 1.00 & $0.81-1.22$ & 0.97 & 1.00 & $0.80-1.23$ & 0.97 \\
\hline Being circumcised means a man can worry less about HIV & 1.01 & $0.89-1.14$ & 0.89 & 1.02 & $0.91-1.16$ & 0.70 \\
\hline If a man is circumcised, he can have more sexual partners & 1.23 & $0.90-1.68$ & 0.20 & 1.21 & $0.89-1.66$ & 0.23 \\
\hline Alcohol consumption & 1.97 & $1.00-3.87$ & 0.05 & 2.30 & $1.45-3.66$ & 0.0004 \\
\hline$<18$ & 1 & ref. & - & 1 & ref. & - \\
\hline $18-20$ & 1.15 & $0.76-1.74$ & 0.46 & 1.09 & $0.73-1.62$ & 0.68 \\
\hline$\geq 21$ & 0.59 & $0.32-1.06$ & 0.08 & 0.71 & $0.38-1.34$ & 0.29 \\
\hline Exchanged money for sex, past 12 months & 1.57 & $0.87-2.82$ & 0.14 & 1.62 & $0.99-2.63$ & 0.05 \\
\hline
\end{tabular}

$\mathrm{RR}$, risk ratio; $\mathrm{Cl}$, confidence intervals; HIV, human immunodeficiency virus; VMMC, voluntary medical male circumcision.

$\dagger$, Estimated from a weighted modified Poisson regression model with weights constructed to adjust for selection bias because of loss to follow-up.

$\$$ Estimated from a weighted modified Poisson regression model with weights constructed to adjust for selection bias because of loss to follow-up and confounding because of the following baseline covariates: age, relationship status, religious affiliation, education, employment and household use of woods as cooking fuel.

$\S$, Responses based on Likert scale (range $0-5$ with 0 and 5 denoting 'strongly disagree' and 'strongly agree', respectively).

\section{Discussion}

In this prospective cohort study of adult men undergoing circumcision within an urban, public-sector clinic in Botswana, we found no evidence of sexual risk compensation in the 3 months following the procedure. Although most participants did not alter their behaviour between baseline and follow-up, amongst those whose behaviour did change, a larger proportion of men reduced, rather than increased, their engagement in risky sexual behaviour as assessed by both the total number and timing of sexual partnerships. Our overall findings are consistent with previous reports from South Africa showing little to no evidence of risk compensation following VMMC. ${ }^{13,14}$

Alcohol consumption was the strongest predictor of subsequent engagement in risky sexual behaviour following VMMC. Previous studies of HIV prevention interventions conducted amongst HIV-negative and HIV-positive persons in the region have yielded similar results. Data from the Sustainable East Africa Research in Community Health (SEARCH) study, a cluster-randomised test-and-treat trial conducted in rural Uganda and Kenya, identified alcohol use as a significant predictor of HIV acquisition in developing an $\mathrm{HIV}$ risk score. ${ }^{25}$ Additionally, in a subset of SEARCH participants also evaluated for tuberculosis acquisition, alcohol users were twice as likely to become infected with tuberculosis compared with non-drinkers, irrespective of HIV status or presence of an infected household contact. ${ }^{26}$ In Botswana, qualitative in-depth interviews conducted with sexually active young adults presenting for care for urogenital complaints cited alcohol use as contributing to inconsistent and/or incorrect condom use. ${ }^{27}$
Most of the existing data around risky sexual behaviour and VMMC relies on cross-sectional surveys (i.e. collected at a single point in time) in which the sexual behaviours of circumcised and uncircumcised men are compared..$^{10,11,12}$ In contrast, we employed a pre-post intervention design in which sexual behaviour was assessed before and after participants underwent VMMC. This is important because men who choose to undergo circumcision may differ systematically from men who do not in ways that are related to risky sexual behaviour but are not easily measured. In our study, each participant served as his own control over time enabling us to control for bias resulting from fixed, systematic differences regardless of whether they are recorded by research staff. A second key strength of our results is the use of ACASI to collect sexual behaviour information. Given the sensitive and private nature of participation in sexual activities, individuals may underestimate or in some cases overestimate engagement in risky sexual behaviour. Indeed, multiple studies have shown that this type of information bias is particularly prevalent in studies on sexual behaviour, which utilise face-to-face interviewing techniques. ${ }^{28,29}$ Misreporting of high-risk sexual behaviour amongst men undergoing VMMC may underestimate prevalence, hindering the effectiveness and safety of circumcision programmes. To mitigate this potential form of information bias, we used ACASI, an interviewing technique that allows the participant to complete the questionnaires privately and without directly sharing their responses with study staff.

Our study is subject to limitations. Firstly, $28 \%$ of men either did not return for follow-up at 3 months post-VMMC and/or did not provide information on risky sexual behaviour postVMMC. Adjustment for selection bias because of this 
sub-optimal retention relies on the assumption that, conditional on a set of observed covariates, men who did and did not return for follow-up are exchangeable, and its appropriateness depends on our ability to identify, measure and control for all covariates associated with loss to followup. Based on subject-matter knowledge, we considered 24 demographic, socio-economic and behavioural covariates and 117 two-way interaction terms in the multivariable model required by the inverse probability weighting procedure. However, as with any observational study, bias because of unmeasured factors is possible.

\section{Conclusion}

Despite these limitations, our results provide reassurance that men undergoing VMMC in Botswana are unlikely to be systematically engaging in risk compensation. Such behaviour, if practised widely, has the potential to undermine the public health benefits of VMMC. Instead, we found that in the 3 months following VMMC, both the overall number and timing of sexual partners were similar to pre-circumcision levels. Botswana's circumcision programme continues to provide HIV prevention through the circumcision procedure itself and is not being jeopardised by the perception of reduced risk of HIV acquisition.

\section{Acknowledgements Competing interests}

All authors declare that they have no financial or personal relationships that may have inappropriately influenced them in writing this article.

\section{Authors' contributions}

L.P.S. and K.E.H. conceived of the presented idea and performed the analysis and wrote the manuscript. S.M. and S.B. verified the analytical methods and edited the final manuscript. J.H.L. supervised the findings of this work and edited the final manuscript. All authors discussed the results and contributed to the final manuscript.

\section{Funding information}

This publication was made possible by grant number U91HA06801 from the U.S. Department of Health and Human Services, Health Resources and Services Administration, HIV / AIDS Bureau's Global Health Systems Branch and grant number UL1TR000423 from the National Center for Research Resources, National Institutes of Health. Its contents are solely the responsibility of the authors and do not necessarily represent the official views of the government.

\section{Data availability statement}

The computing code and an anonymised analytic data set used to produce the results contained in this manuscript are available upon request from the corresponding author.

\section{Disclaimer}

Views expressed in the submitted article are our own and do not necessarily reflect the official policy or position of our institutions or funders.

\section{References}

1. Auvert B, Taljaard D, Lagarde E, Sobngwi Tambekou J, Sitta R, Puren A. Randomized controlled intervention trial of male circumcision for reduction of HIV infection risk: The ANRS 1265 trial. PLoS Med. 2005;2(11):e298. https://doi.org/10.1371/ journal.pmed.0020298

2. Gray RH, Kigozi G, Serwadda D, et al. Male circumcision for HIV prevention in men in Rakai, Uganda: A randomised trial. Lancet. 2007;369(9562):657-666. https:// doi.org/10.1016/S0140-6736(07)60313-4

3. Bailey RC, Moses S, Parker CB, et al. Male circumcision for HIV prevention in young men in Kisumu, Kenya: A randomised controlled trial. Lancet. 2007:369(9562): 643-656. https://doi.org/10.1016/S0140-6736(07)60312-2

4. Auvert B, Marseille E, Korenromp EL, et al. Estimating the resources needed and savings anticipated from roll-out of adult male circumcision in sub-Saharan Africa. PLoS One. 2008;3(8):e2679. https://doi.org/10.1371/journal.pone.0002679

5. Podder CN, Sharomi O, Gumel AB, Moses S. To cut or not to cut: A modeling approach for assessing the role of male circumcision in HIV control. Bull Math Biol. 2007;69(8):2447-2466. https://doi.org/10.1007/s11538-007-9226-9

6. Nagelkerke NJ, Moses S, De Vlas SJ, Bailey RC. Modelling the public health impact of male circumcision for HIV prevention in high prevalence areas in Africa. BMC Infect Dis. 2007;7:16. https://doi.org/10.1186/1471-2334-7-16

7. UNAIDS. Country factsheets: Botswana [homepage on the Internet]. 2017 [cited 2018 Aug 23]. Available from: http://www.unaids.org/en/regionscountries/ countries/botswana

8. Williams BG, Gupta S, Wollmers M, Granich R. The impact and cost of ending AIDS in Botswana. Lancet HIV. 2016;3(9):E409. https://doi.org/10.1016/S2352-3018 (16)30116-3

9. Bollinger LA, Stover J, Musuka G, Fidzani B, Moeti T, Busang L. The cost and impact of male circumcision on HIV/AIDS in Botswana. J Int AIDS Soc. 2009;12(1):7. https://doi.org/10.1186/1758-2652-12-7

10. Govender K, George G, Beckett S, Montague C, Frohlich J. Risk compensation following medical male circumcision: Results from a 1-year prospective cohort study of young school-going men in KwaZulu-Natal, South Africa. Int J Behav Med. 2018;25:123-130. https://doi.org/10.1007/s12529-017-9673-0

11. Kankaka EN, Ssekasanvu J, Prodger J, et al. Sexual risk behaviors following circumcision among HIV-positive men in Rakai, Uganda. AIDS Care. 2018; 30(8):990-996. https://doi.org/10.1080/09540121.2018.1437253

12. Westercamp M, Jaoko W, Mehta S, Abuor P, Siambe P, Bailey R. Changes in male circumcision prevalence and risk compensation in the Kisumu, Kenya population 2008-2013. J Acquir Immune Defic Syndr. 2017;74(2):e30-e37. https://doi. org/10.1097/QAI.0000000000001180

13. Mukudu H, Dietrich J, Otwombe K, et al. Voluntary medical male circumcision (VMMC) for prevention of heterosexual transmission of HIV and risk compensation in adult males in Soweto: Findings from a programmatic setting. PLoS One. 2019;14(3):e0213571. https://doi.org/10.1371/journal.pone.0213571

14. Ortblad KF, Harling G, Chimbindi N, Tanser F, Salomon JA, Barnighausen T. Does incident circumcision lead to risk compensation? Evidence from a population cohort in KwaZulu-Natal, South Africa. J Acquir Immune Defic Syndr. 2019; 80(3):269-275. https://doi.org/10.1097/QAl.0000000000001912

15. Makhema J, Wirth KE, Pretorius Holme M, et al. Universal testing, expanded treatment, and incidence of HIV infection in Botswana. N Engl J Med. 2019;381:230-242. https://doi.org/10.1056/NEJMoa1812281

16. Ministry of Health. Safe male circumcision: Additional strategy for HIV prevention: A national strategy. Gaborone: Ministry of Health; 2010.

17. Connor RJ. Sample size for testing differences in proportions for the pairedsample design. Biometrics. 1987;43(1):207-211. https://doi.org/10.2307/2531961

18. Ho-Foster A, Laetsang D, Masisi M, et al. Gender-specific patterns of multiple concurrent sexual partnerships: A national cross-sectional survey in Botswana. AIDS Care. 2010;22(8):1006-1011. https://doi.org/10.1080/09540121003758598

19. Eaton LA, Cain DN, Agrawal A, Jooste S, Udemans N, Klichman SC. The influence of male circumcision for HIV prevention on sexual behavior among traditionally circumcised men in Cape Town, South Africa. Int J STD AIDS. 2011;22(11): 674-679. https://doi.org/10.1258/ijsa.2011.011006

20. Harris PA, Taylor R, Thielke, JP, Payne J, Gonzalez N, Conde JG. Research electronic data capture (REDCap) - A metadata-driven methodology and workflow process for providing translational research informatics support. J Biomed Inform. 2009;42(2):377-381. https://doi.org/10.1016/j.jbi.2008.08.010

21. UNAIDS reference group on estimates, modelling and projections: Working group on measuring concurrent sexual partnerships. HIV: Consensus indicators are needed for concurrency. Lancet. 2009;375(9715):621-622. https://doi.org/ 10.1016/S0140-6736(09)62040-7 
22. Hernan MA, Hernandez-Díaz S, Robins JM. A structural approach to selection bias. Epidemiology. 2004;15(5):615-625. https://doi.org/10.1097/01.ede.0000135174. 63482.43

23. Scharfstein DO, Rotnitzky A, Robins JM. Adjusting for nonignorable drop-out using semiparametric nonresponse models. J Am Stat Assoc. 1999;94(448):1096-1120. https://doi.org/10.2307/2669930

24. Robins JM, Rotnitzky A, Zhao LP. Estimation of regression coefficients when some regressors are not always observed. J Am Stat Assoc. 1994;89(427):846-866. https://doi.org/10.1080/01621459.1994.10476818

25. Balzer LB, Havlir DV, Kamya MR, et al. Machine learning to identify persons at high-risk of human immunodeficiency virus acquisition in rural Kenya and Uganda. Clin Infect Dis. 2019; e1-e8.
26. Puryear SB, Mucunguzi A, Balzer LB, et al. Alcohol use is associated with incident TB infection in HIV+ and HIV- Ugandan adults. Paper presented at: Conference on retroviruses and opportunistic infections; 2020 Aug 3 - Nov 3; Boston, MA

27. Kanda L, Mash R. Reasons for inconsistent condom use by young adults in Mahalapye, Botswana. Afr J Prim Health Care Fam Med. 2018;10(1):a1492. https://doi.org/10.4102/phcfm.v10i1.1492

28. Des Jarlais DC, Paone D, Milliken J, et al. Audio-computer interviewing to measure risk behaviour for HIV among injecting drug users: A quasi-randomised trial. Lancet. 1999;353(9165):1657-1661. https://doi.org/10.1016/S0140-6736(98)07026-3

29. Tideman RL, Chen MY, Pitts MK, Ginige S, Slaney M, Fairley CK. A randomised controlled trial comparing computer-assisted with face-to-face sexual history taking in a clinical setting. Sex Transm Infect. 2007;83(1):52-56. https://doi. org/10.1136/sti.2006.020776 